\title{
Theory of the prompt emission of Gamma-Ray Bursts
}

\section{Davide Lazzati*}

Department of Physics, NC State University, Raleigh, NC 27695-8202

E-mail: davide_lazzatiencsu.edu

\begin{abstract}
Since their discovery more than 40 years ago the origin of the prompt emission of gamma-ray bursts has been highly controversial. For decades, the standard emission model has been considered synchrotron radiation from shock-heated electrons. More recently, theoretical work in support of a photospheric origin of the bursts' prompt spectrum has accumulated, bringing Comptonized thermal radiation to the front line as an alternative model to the standard scenario. In this review I will discuss the observational constraints and the pros and cons that each radiation mechanism has. I will conclude by emphasizing that the GRB spectrum is likely the result of the superposition of all radiation mechanisms, with photospheric emission playing a leading role in defining the domimant photon energy.
\end{abstract}

Gamma-Ray Bursts 2012 Conference-GRB2012,

May 07-11, 2012

Munich, Germany

${ }^{*}$ Speaker. 


\section{Observational constraints}

The first thing a theorist needs to remember when attempting to find a model to explain the origin of the GRB prompt radiation spectrum is what is in the data. The GRB prompt emission is characterized by various observational constraints.

1. Non-thermal. The prompt radiation is non-thermal. The integrated spectrum has power-law tails at both low- and high-frequency (e.g., Kaneko et al. 2006).

2. Peak frequency. In most cases, the low- and high-frequency slopes are such that they define a peak between them in the $v F(v)$ spectrum. This photon energy ranges between a few tens of keV up to several MeV (e.g., Kaneko et al. 2006).

3. Typical power-law indices. The average low-frequency power-law index is $F(v) \propto v^{0}$, while the average high-frequency index is $F(v) \propto v^{-1.5}$ (e.g., Kaneko et al. 2006).

4. Very high-frequency emission. In some cases the high-frequency spectrum extends to $\mathrm{GeV}$ photons (Abdo et al. 2009).

5. Spectral evolution. GRBs are divided between tracking and hard-to-soft. Tracking GRBs have peak photon energy that tracks the burst luminosity, while hard-to-soft bursts have a monotonic decrease of the peak energy through the prompt phase.

6. Variability. Both indices vary during the burst. The low-frequency index $(\alpha)$ can be much steeper than the average at the beginning of the burst. Cases with $F(v) \propto v^{2}$ have been recorded (Ghirlanda et al. 2003; Ryde 2005).

7. Efficiency. When it is possible to measure the efficiency of converting the outflow total energy into radiation, the prompt emission efficiency is found to be large, typically around $50 \%$ (Zhang et al. 2007).

8. Asymmetry. GRB pulses are typically asymmetric, with rise times that are faster than decay times (e.g., Norris et al. 1996).

9. Amati correlation. The isotropic equivalent energy of long-duration bursts and their timeintegrated peak frequencies are positively correlated (Amati et al. 2002).

10. Yonetoku correlation. A positive correlation also exists between the isotropic equivalent peak luminosity and the peak frequency. This correlation also holds for short bursts (Yonetoku et al. 2004).

11. Lorentz factor correlations. Correlations have been found between the Lorentz factor of the outflow and the energy, peak luminosity, and peak frequency of long duration GRBs (Liang et al. 2010; Ghirlanda et al. 2012). All these correlations are positive.

Besides these observational constraints, one should always remember that the prompt emission radiation mechanism cannot be investigated independently of the related issues of the jet composition and the dissipation mechanism. 


\section{The synchrotron shock model}

Traditionally, the synchrotron shock model has been considered to be the best candidate to explain GRB prompt emission observations (e.g., Piran 1999). Within this framework, the GRB outflow is supposed to be generated by an unsteady engine of radius $R_{0}$, releasing shells of material with different Lorentz factor $\Gamma$. At the internal shock radius $R_{I S} \sim R_{0} \Gamma^{2}$ the shells collide producing mildly relativistic shocks in the outflow frame. Electrons are accelerated in a non-thermal population at the shock front and a tangled magnetic field is generated. Radiation is produced through the synchrotron process. At the internal shock radius the fireball is optically thin and the radiation escapes to infinity.

The advantage of the synchrotron shock model is that of naturally explaining the non-thermal appearance of the busts' spectra. In addition, the typical frequency can be accounted for with values for the unknown parameters that are within the acceptable range. However, the synchrotron shock model has several issues with the explanation of some of the bursts observations listed above. First, the synchrotron spectrum cannot be harder, in the low-frequency range, than $F(v) \propto v^{1 / 3}$. However, bursts with harder spectra have been observed (Preece et al. 1998). In addition, it can be shown that the electrons are expected to be in the fast-cooling regime, causing an even softer typical spectrum $F(v) \propto v^{-1 / 2}$ at low-frequency (Ghisellini et al. 1999). Second the synchrotron shock model is plagued by small efficiency. The internal shocks themselves cannot be more efficient than $\sim 10 \%$ (Lazzati et al. 1999), since they dissipate only the relative kinetic energy of the outflow and not its bulk. In addition, only up to $1 / 3$ of the dissipated energy can be given to the electrons, so that the overall process is at most a few per cent efficient. Third the synchrotron shock model predicts that the peak frequency of the spectrum is negatively correlated with the Lorentz factor of the outflow. In other words, faster outflows should produce bursts with lower peak frequency. This constraint arises from the fact that faster outflows produce internal shocks at larger radii and therefore generate magnetic fields of smaller intensity and eventually smaller peak frequencies (see, e.g., Eq. 3 in Ghisellini et al. 1999).

\section{Photospheric models}

The main characteristic of photospheric models is not that of identifying a specific radiation mechanism but that of assuming that the radiation is formed in the highly optically thick part of the outflow, advected with it, shaped at moderate optical depth, and released at the photosphere (optical depth of 1). Since GRB outflows are optically thick when they form (even if baryon free, they would immediately form a pair plasma that is optically thick) some photospheric emission is part of any GRB model. However, what is important, and subject of current research, is what fraction of the energy is released in photons at the photosphere and what is the shape of their spectrum. The photospheric spectrum that is released by a dissipationless outflow expanding spherically (or conically) is somewhat close to a Planck spectrum, but has a softer low energy spectrum (Goodman 1986; Beloborodov 2011). That is due to the fact that the spectrum is produced when the outflow is highly opaque and the expansion of a fireball (or conical jet) accelerated by its own internal pressure conserves the photon number. 


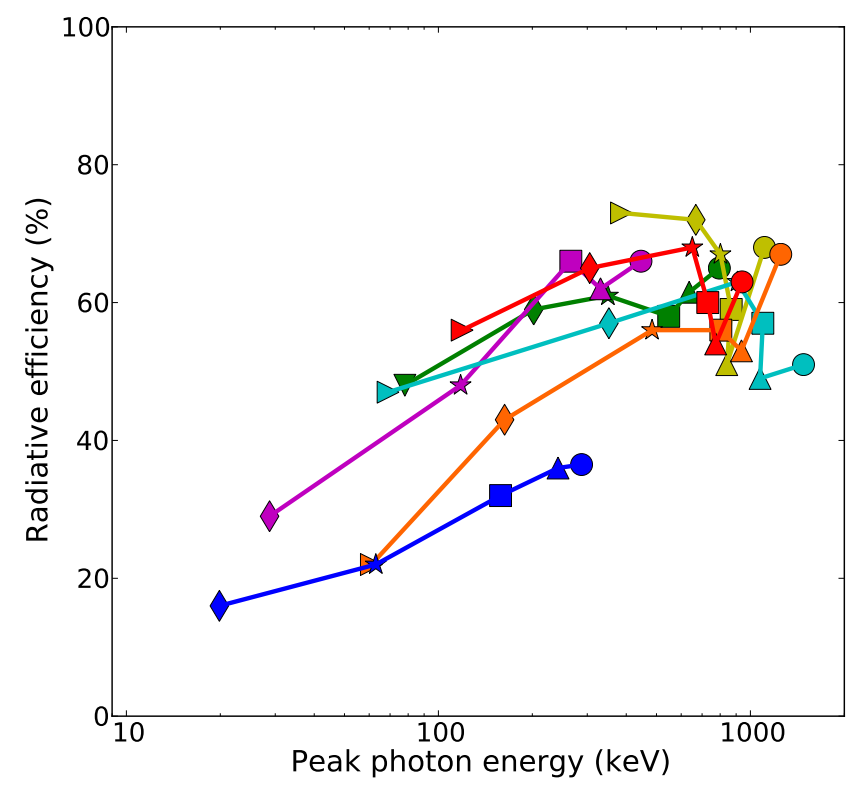

Figure 1: Efficiency of GRB jet photospheres from numerical simulations of hydrodynamic jets in massive progenitor stars (Lazzati et al. submitted).

Sub-photospheric dissipation The interaction between the leptons and the photons in the optically thick part of a GRB jet happens via inverse Compton interactions. The typical frequency of photons is set at optical depth of several tens (Giannios 2012). From there to the photosphere (in the range $50 \lesssim \tau<1$ ) photons cannot be cooled by the inverse Compton process, but they can be heated by direct Compton scattering off hot electrons. The main consequence of this is that if any dissipation is present at moderate optical depth, and this dissipation creates a population of electron out of thermal equilibrium with the radiation, the spectrum released at the photosphere is highly non-thermal, with a prominent high-frequency power-law tail (Giannios 2006; Pe'er et al. 2006; Beloborodov 2010; Lazzati \& Begelman 2010). The radiation coming from dissipative photospheres is therefore in agreement with the non-thermal shape of the prompt emission, at least in the high-frequency range up to $h v=511 \Gamma \mathrm{keV}$.

GeV Photons Within the photospheric scenario, photons with energy $h v>50 \Gamma_{2} \mathrm{MeV}^{1}$ are absorbed due to photon-photon collisions to produce electron-positron pairs. As a consequence, the $\mathrm{GeV}$ emission observed in several burst by Fermi (e.g., Abdo et al. 2009) must have a different origin. One possibility is that the $\mathrm{GeV}$ emission is from the external shock, the other is that it is from a prompt component produced at larger radius than the photosphere.

The low-frequency range Explaining the fact that only a few bursts have steep $F(v) \propto v^{2}$ lowfrequency spectra is probably the most challenging aspect of the photospheric theory. Since the typical GRB prompt spectra have more photons at low frequency than at the peak frequency, it is not possible to explain the flat low-frequency slope by downscattering. The most likely explanation

\footnotetext{
${ }^{1}$ Numerical simulations show that the actual absorption happens at a higher frequency (Beloborodov 2010).
} 


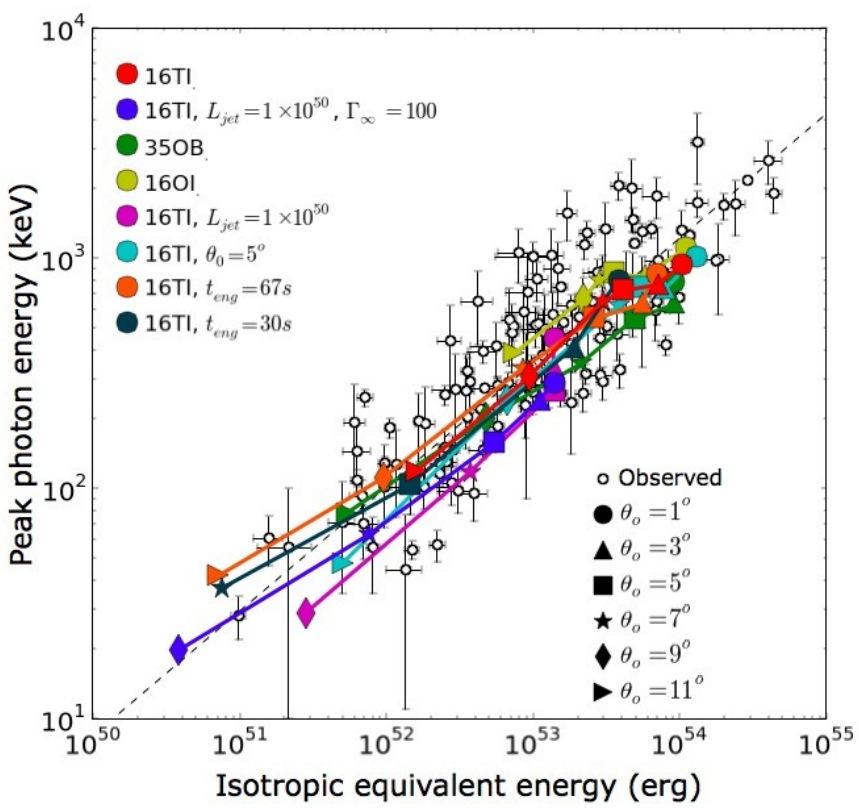

Figure 2: Comparison between the observed Amati correlation (black points with error bars) and the result of numerical simulations (each color corresponding to a different jet-progenitor system).

is that there is an additional radiation component that is produced at the photosphere or at larger radii. This component should be small in terms of the energy budget and have a flat spectrum dominated by low-frequency emission. The only viable model proposed so far is based on an extra synchrotron component (Vurm et al. 2011). In their model, only very low-frequency photons are produced at radii larger than the photosphere.

Efficiency Among the positive features of the photospheric model with respect to the synchrotron shock model is that is has high efficiency (Lazzati et al. 2009, Figure 1). The high efficiency is not due to a highly efficient dissipation but to the fact that the outflow does not accelerate to more than half its asymptotic Lorentz factor. As a consequence, approximately half of the outflow energy is released as radiation at the photosphere. Note that the model predicts a correlation between the burst peak frequency and the radiative efficiency of the prompt phase.

Correlations Despite being discovered more than a decade ago, the Amati correlation (Amati et al. 2002) is still unexplained and subject to continuous debate on whether it's real or affected by selection effects (see various other contributions to these proceedings). Numerical simulations can be used to compute the isotropic equivalent energy and the temperature of the radiation at the photosphere. Results for hydrodynamic jets are in excellent agreement with the observations (Figure 2; Lazzati et al. submitted). Simulations performed with different progenitors and different jet initial conditions (different colors in the Figure) have consistently returned value of the peak frequency in agreement with the observations. Analogous results are obtained with the Lorentz factor-peak frequency correlation (Figure 3). 


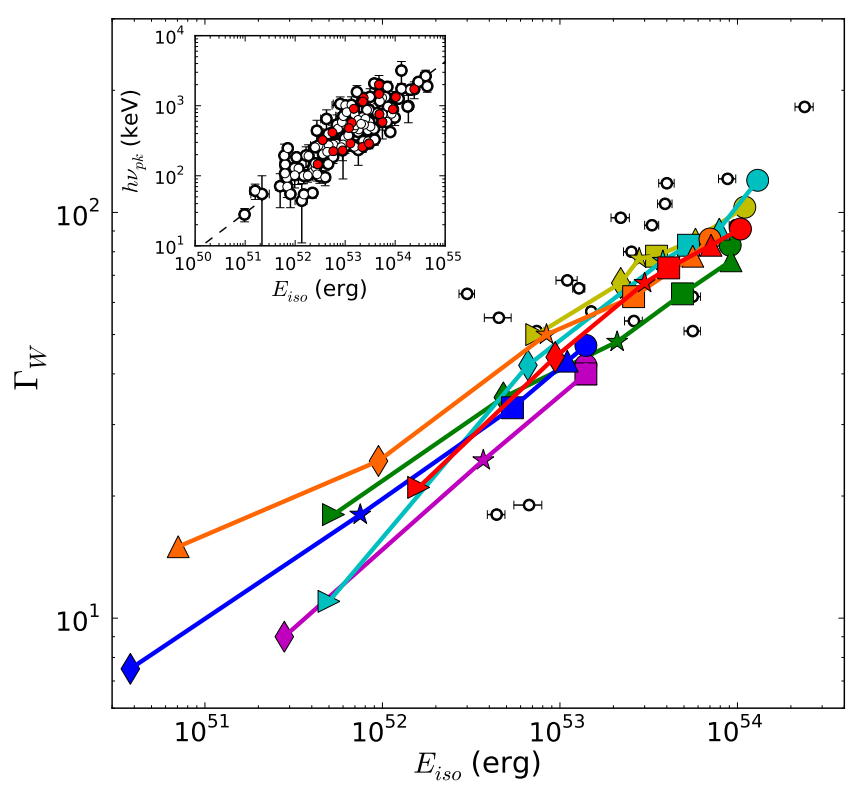

Figure 3: Comparison between the observed $\Gamma-h v_{\mathrm{pk}}$ correlation (black points with error bars) and the result of numerical simulations (each color corresponding to a different jet-progenitor system).

\section{Discussion and conclusions}

The prompt emission of GRBs has puzzled astrophysicists for several decades and it's therefore unlikely that it can be explained by a single emission mechanism. After being considered the best candidate for a few decades, synchrotron radiation is now being considered only one part of a bigger picture in which photospheric radiation, inverse Compton, and synchrotron play an important role. This new picture is far from being well defined and understood, but seem more promising to be able to explain all the features and the diversity of the observations.

The first important change with respect to the old standard is that there is not an emission radius where the whole spectrum is produced. In the photospheric scenario the bulk of the radiation is produced at very small radii, where the jet is optically thick. It is then advected and cooled by the jet leptons. When the subphotospheric region is reached, non-thermal high-frequency tails are added by inverse Compton scattering off energized electrons. The dissipation can be produced by different mechanisms, recollimation shocks, internal shocks, magnetic reconnection, and/or internal proton-proton collisions in a neutronized outflow. The result is fairly independent of the details. At the photosphere or at larger radii an additional component must be invoked to explain the soft low-energy tails, and another component may be required for bursts with $\mathrm{GeV}$ emission. Indeed both the soft low-frequency tails and the GeV photons seem to be systematically delayed, consistent with the requirement of an extra component at lager radius.

Among the unclear aspects of this emerging scenario is the role of short-duration bursts. The characteristics of their prompt emission and the fact that they line up with long GRBs along the Yonetoku correlation suggest that they have the same emission mechanism as long bursts. However, they do not have massive star progenitors. Whether the similarities are just a coincidence or have 
a physical meaning that we haven't understood yet is probably the aspect of this theory that most desperately needs attention.

\section{References}

[1] A. A. Abdo et al., Fermi Observations of High-Energy Gamma-Ray Emission from GRB 080916C, Science 323 (2009) 1688.

[2] L. Amati et al., Intrinsic spectra and energetics of BeppoSAX Gamma-Ray Bursts with known redshifts, A\&A 390 (2002) 81 [astro-ph/0205230].

[3] A. Beloborodov, Collisional mechanism for gamma-ray burst emission, MNRAS 407 (2010) 1033 [arXiv:0907.0732].

[4] A. Beloborodov, Radiative Transfer in Ultrarelativistic Outflows, ApJ 737 (2011) 68 [arXiv:1011.6005].

[5] G. Ghirlanda, A. Celotti, and G. Ghisellini, Extremely hard GRB spectra prune down the forest of emission models, A\&A 406 (2003) 879 [astro-ph/0210693].

[6] G. Ghirlanda et al., Gamma-ray bursts in the comoving frame, MNRAS 420 (2012) 483 [arXiv:1107.4096].

[7] G. Ghisellini, A. Celotti, and D. Lazzati, Constraints on the emission mechanisms of gamma-ray bursts, MNRAS 313 (1999) L1 [astro-ph/9912461].

[8] D. Giannios, Prompt emission spectra from the photosphere of a GRB, A\&A 457 (2006) 763 [astro-ph/0602397].

[9] D. Giannios, The peak energy of dissipative gamma-ray burst photospheres, MNRAS 422 (2012) 3092 [arXiv:1111.4258].

[10] J. Goodman, Are gamma-ray bursts optically thick?, ApJ 308 (1986) L47.

[11] Y. Kaneko et al., The Complete Spectral Catalog of Bright BATSE Gamma-Ray Bursts, ApJS 166 (2006) 298 [astro-ph/0601188].

[12] D. Lazzati, G. Ghisellini, and A. Celotti, Constraints on the bulk Lorentz factor in the internal shock scenario for gamma-ray bursts, MNRAS 309 (1999) L13 [astro-ph/9907070].

[13] D. Lazzati, B. J. Morsony, and M. C. Begelman, Very High Efficiency Photospheric Emission in Long-Duration $\gamma$-Ray Bursts, ApJ 700 (2009) L47 [arXiv:0904.2779].

[14] D. Lazzati and M. C. Begelman, Non-thermal Emission from the Photospheres of Gamma-ray Burst Outflows. I. High-Frequency Tails, ApJ 725 (2010) 1137 [arXiv:1005.4704].

[15] E. Liang et al., Constraining Gamma-ray Burst Initial Lorentz Factor with the Afterglow Onset Feature and Discovery of a Tight $\Gamma_{0}-E_{\gamma, \text { iso }}$ Correlation, ApJ 725 (2010) 2209 [arXiv:0912.4800].

[16] J. P. Norris et al., Attributes of Pulses in Long Bright Gamma-Ray Bursts, ApJ 459 (1996) 393.

[17] A. Pe'er, P. Meszaros, and M. J. Rees, The Observable Effects of a Photospheric Component on GRB and XRF Prompt Emission Spectrum, ApJ 642 (2006) 995 [astro-ph/0510114].

[18] T. Piran, Gamma-ray bursts and the fireball model, Physics Reports 314 (1999) 575 [astro-ph/9810256].

[19] R. D. Preece et al., The Synchrotron Shock Model Confronts a "Line of Death" in the BATSE Gamma-Ray Burst Data, ApJ 506 (1998) L23 [astro-ph/9808184]. 
[20] F. Ryde, Is Thermal Emission in Gamma-Ray Bursts Ubiquitous?, ApJ 625 (2005) L95 [astro-ph/0504450].

[21] I. Vurm, A. M. Beloborodov, and J. Poutanen, Gamma-Ray Bursts from Magnetized Collisionally Heated Jets, ApJ 738 (2011) 77 [arXiv:1104.0394].

[22] D. Yonetoku et al., Gamma-Ray Burst Formation Rate Inferred from the Spectral Peak Energy-Peak Luminosity Relation, ApJ 609 (2004) 935 [astro-ph/0309217].

[23] B. Zhang et al., GRB Radiative Efficiencies Derived from the Swift Data: GRBs versus XRFs, Long versus Short, ApJ 655 (2007) 989 [astro-ph/0610177]. 